\title{
QUANTUM INFORMATION AND THE PROBLEM OF TIME
}

\section{GEORGE SVETLICHNY}

Communicated by Ivaïlo M. Mladenov

\begin{abstract}
In many cases tensor universality implies that multi-partite quantumstate processing is determined by what happens in totally disentangled cases. In independent systems relative time direction for the parts is arbitrary. This hints that time may be linked to entanglement and measurements and that there may be a measurement-only version of quantum mechanics. One-way quantum computation suggests that this may be possible.
\end{abstract}

\section{Introduction}

We have recently proved a theorem implying that under certain conditions quantum state processing in entangled systems is uniquely determined by processing in totally disentangles systems [3]. In totally disentangles systems time direction is arbitrary in the independent parts so one can ask: does time arise from entanglement and measurements? In particular, can unitary time evolution be reduced to entanglement and measurement? This is the reverse side of the quantummechanical measurement problem. Instead of "How do measurements happen?" we ask "How does unitary time evolution happen?". In other words: is there a measurement-only model of quantum mechanics? We argue that "yes" is a plausible answer.

We begin by illustrating the theorem in simple cases, including teleportation.

\section{Tensor Universality and Quantum-state Processing}

Consider subjecting a multipartite Heisenberg quantum state $\Phi \in \mathcal{H}_{1} \otimes \cdots \otimes \mathcal{H}_{n}$ to $m$ successive observations, described by self-adjoint opertors $A_{1}, \ldots, A_{m}$. Each $A_{j}$ acts in a subproduct of $\mathcal{H}=\mathcal{H}_{1} \otimes \cdots \otimes \mathcal{H}_{n}$ where we assume it is nondegenerate. Assume for simplicity that each $A_{i}$ is a bipartite operator. 
In a given run of the experiment, the state transforms to $\Psi=P_{m} \cdots P_{1} \Phi$ where each $P_{j}$ is a rank-one spectral projection of $A_{j}$ in a bipartite subproduct of $\mathcal{H}$.

Given a tensor product Hilbert space $\mathcal{H} \otimes \mathcal{K}$ and a vector $\Omega \in \mathcal{H} \otimes \mathcal{K}$ one can uniquely define two linear maps $g_{\Omega}: \mathcal{H} \rightarrow \mathcal{K}^{*}$ and $f_{\Omega}: \mathcal{H}^{*} \rightarrow \mathcal{K}$ (the asterisk denotes the dual space) which in case $\Omega=\alpha \otimes \beta$ act as: $g_{\Omega}:|\phi\rangle \mapsto\langle\alpha \mid \phi\rangle\langle\beta|$ and $f_{\Omega}:\langle\phi|\mapsto\langle\phi \mid \alpha\rangle| \beta\rangle$.

Consider a particular example in $\mathcal{H}_{1} \otimes \mathcal{H}_{2} \otimes \mathcal{H}_{3} \otimes \mathcal{H}_{4} \otimes \mathcal{H}_{5}$ given by $\Psi=$ $P_{4}^{(34)} P_{3}^{(12)} P_{2}^{(23)} P_{1}^{(45)} \Phi$ where the superscripts indicate on which bipartite subproduct the projections act. Let $P_{i}=\left|\Omega_{i}\right\rangle\left\langle\Omega_{i}\right|$. Coeke's theorem [2], a particular case of our theorem and presented here in a slightly modified form, now states that if the initial state is $\phi_{1}^{\text {in }} \otimes \Phi_{2345}^{\text {in }} \in \mathcal{H}_{1} \otimes\left(\mathcal{H}_{2} \otimes \mathcal{H}_{3} \otimes \mathcal{H}_{4} \otimes \mathcal{H}_{5}\right)$, then the final state is $\Phi_{1234}^{\text {out }} \otimes \phi_{5}^{\text {out }} \in\left(\mathcal{H}_{1} \otimes \mathcal{H}_{2} \otimes \mathcal{H}_{3} \otimes \mathcal{H}_{4}\right) \otimes \mathcal{H}_{5}$, where

$$
\phi_{5}^{\text {out }}=g_{\Omega_{1}} \circ f_{\Omega_{4}} \circ g_{\Omega_{2}} \circ f_{\Omega_{3}}\left(\phi_{1}^{\text {in }}\right) .
$$

Pictorially we have Fig. 1 where we have put in arrows to indicate the apparent direction of "quantum information flow". We see that the processing order, given by the composition of maps in (1), is not the temporal order and "quantum information" switches between "forward" and "backward time flow". We consider as metaphorical all the expressions in quotes in this paragraph.

It is quite remarkable that Coecke's theorem can be proved by considering only the case in which all relevant states are disentangled. To do this we must modify the state transformer by replacing each projector $P_{\Omega}$ by a general rank-one operator $\left.Q_{\Lambda, \Omega}=|\Lambda\rangle\langle\Omega|=\Lambda \otimes \Omega\right\rfloor$. where the floor symbol "」" denotes the partial inner product defined by $\Omega\rfloor \alpha \otimes \beta=(\Omega, \alpha) \beta$.

Consider now the transformation:

$$
\left.\left.\Psi=Q_{m} \cdots Q_{1} \Phi=\Lambda_{m} \otimes \Omega_{m}\right\rfloor \otimes \cdots \Lambda_{1} \otimes \Omega_{1}\right\rfloor \Phi .
$$

This is anti-linear in each $\Omega_{j}$ and linear in each $\Lambda_{j}$ and in each factor of $\Phi$ if disentangled from the rest.

As an example take $\Psi=Q_{3}^{(23)} Q_{2}^{(12)} Q_{1}^{(23)} \Phi$. We now disentangle everything. That is, $\Phi=\phi_{1} \otimes \phi_{2} \otimes \phi_{3}, \Lambda_{j}=\mu_{j} \otimes \nu_{j}$, and $\Omega_{j}=\sigma_{j} \otimes \tau_{j}$. Pictorially we get Fig. 2 which depicts three completely independent quantum processes (say, on Mars, Earth, and Venus).

The outcome state is:

$$
\left\{\left(\sigma_{2}, \phi_{1}\right) \mu_{2}\right\} \otimes\left\{\left(\sigma_{1}, \phi_{2}\right)\left(\tau_{2}, \mu_{1}\right)\left(\sigma_{3}, \nu_{2}\right) \mu_{3}\right\} \otimes\left\{\left(\tau_{1}, \phi_{3}\right)\left(\tau_{3}, \nu_{1}\right) \nu_{3}\right\} .
$$




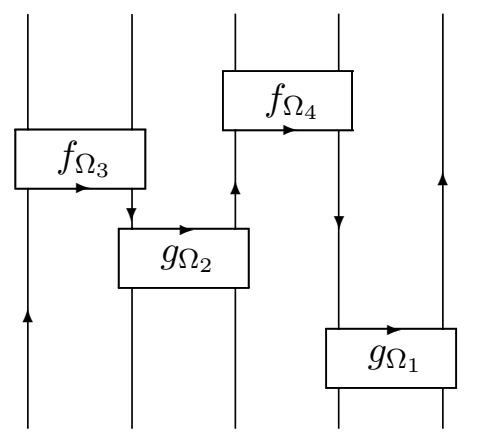

Figure 1. Processing order given by arrows

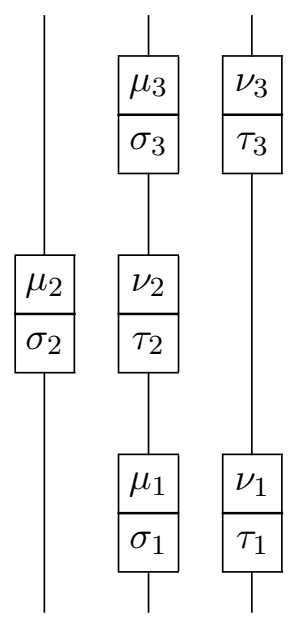

Figure 2. Totally disentangled case

We rewrite the outcome state by moving the scalar inner product around:

$$
\left\{\left(\sigma_{2}, \phi_{1}\right)\left(\tau_{2}, \mu_{1}\right)\left(\tau_{3}, \nu_{1}\right)\left(\sigma_{3}, \nu_{2}\right) \mu_{2}\right\} \otimes\left\{\mu_{3}\right\} \otimes\left\{\nu_{3}\left(\sigma_{1}, \phi_{2}\right)\left(\tau_{1}, \phi_{3}\right)\right\} .
$$

This last expression can be written as ("op" means reverse order in $\otimes$ )

$$
\left.g_{\Lambda_{2}}^{\text {op }} \circ f_{\Omega_{3}}^{\text {op }} \circ g_{\Lambda_{1}} \circ f_{\Omega_{2}}\left(\phi_{1}\right) \otimes\left(\Lambda_{3} \otimes \Omega_{1}\right\rfloor \Phi_{23}\right) .
$$

This is also anti-linear in each $\Omega_{j}$ and linear in each $\Lambda_{j}$ and in each factor of $\Phi$ if disentangled from the rest.

Tensor universality says that two linear (or anti-linear) maps on $\mathcal{H} \otimes \mathcal{K}$ that coincide on product states are identical. Thus (2) is the output state in all cases of $\Phi$ being of the form $\phi_{1} \otimes \Phi_{23}$ and the $\Omega_{i}$ and $\Lambda_{i}$ completely arbitrary. Assuming $\Lambda_{i}=\Omega_{i}$ this is a particular case of Coecke's theorem.

Let now $\mathcal{H}_{1} \otimes \mathcal{H}_{2} \otimes \mathcal{H}_{3}$ be a three-qubit Hilbert space. Alice has access to $\mathcal{H}_{1} \otimes \mathcal{H}_{2}$ and Bob to $\mathcal{H}_{3}$. Let $\Theta=(|0\rangle|1\rangle-|1\rangle|0\rangle) / \sqrt{2}$, then $g_{\Theta}^{(12)} \circ f_{\Theta}^{(23)} \phi_{1}=-\frac{1}{2} \phi_{1}$ and so this is an instance of teleportation if by $\Theta^{(23)}$ we mean an entangled pair shared by Alice and Bob (produced, say, by a third party and invariable), and $\Theta^{(12)}$ is an element of a basis of a two-qubit measurement by Alice. For other instances of Alice's projection on a measurement eigenbasis element, she sends classical information to Bob who then adjusts his qubit by a unitary transformation to achieve teleportation in all cases. But this teleportation behavior is a logical consequence of the behavior when all the relevant states are completely disentangled. Thus 
from independent quantum processes on Mars, Earth and Venus, we can deduce that one can teleport a qubit from Rio de Janeiro to Kiev if one has good enough optical cable and a source of entangled photons on the Canary Islands.

In totally disentangles systems time direction is arbitrary in the independent parts. Teleportation, barring the Everett and other measurement-less interpretations, is clearly not a time reversible process (there's classical communication involved). So how can behavior of disentangled systems, with weak global temporal structure, imply that of systems with stronger temporal structure. Once again one raises the question as to where time comes from, in particular, does it arise from entanglement and measurements? One-way quantum computation [1] seems to suggest that such an interpretation is plausible.

\section{One-way Quantum Computation and Measurement-only Quantum Mechanics}

In one-way quantum computation, quantum gates are simulated by measurements and classical communication, modulo an initial entanglement. Since one can approximate any unitary by a quantum gate circuit, one can in principle simulate unitary time evolution through measurements and classical communication. This would bring us closer to the view that the world is made exclusively of "events" (measurements). Opposite is the Everett view where there are no events, and in between is the dualistic Copenhagen view with events and unitary evolution, which is event-less. One can thus ask: is the universe a one-way quantum computer? To get some idea of this question, consider Fig. 3 as a typical one-way quantum computation.

In this picture real time runs upward just as in the previous diagrams. Upward lines are qubit Hilbert spaces. Simulated time runs horizontally. Thick lines denote entanglement, and what is being simulated is a quantum gate, say CNOT.

To illustrate the essence of one-way computation let us consider how one would simulate the unitary map $U$ acting on a vector $\psi=\alpha|0\rangle+\beta|1\rangle$ given by $U$ : $\alpha|0\rangle+\beta|1\rangle \mapsto \alpha|0\rangle+\mathrm{e}^{\mathrm{i} \phi} \beta|1\rangle$. One has to:

1) Prepare the entangled state $\alpha|0\rangle|0\rangle+\beta|1\rangle|1\rangle$

2) Measure the first qubit in the basis $\eta_{ \pm}=\left(|0\rangle \pm \mathrm{e}^{\mathrm{i} \phi}|1\rangle\right) / \sqrt{2}$.

The resulting state after this measurement is $\eta_{ \pm} \otimes\left(\alpha|0\rangle \pm \mathrm{e}^{\mathrm{i} \phi}|1\rangle\right)=\eta_{ \pm} \otimes \psi_{ \pm}$ according to whether the measurement projected the first qubit to $\eta_{+}$or $\eta_{-}$. Now the second tensor factor in this state is the desired state $U \psi$ in case of the $\eta_{+}$ 


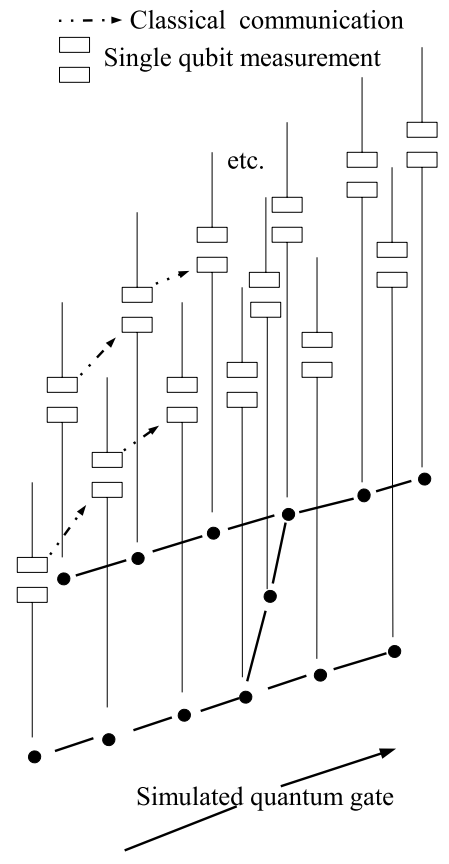

Figure 3. One-way quantum processing

result, but in the case of $\eta_{-}$it is not. Thus we cannot really implement unitary maps by measurements and classical communication, but that is not the point. We can simulate their action if by simulation we mean that we also include the result of the final measurement. In quantum computation one makes a final query of the system to get the result. So what we really want is not to be able to produce $U \psi$ but to reproduce the transition probabilities $|\langle\omega|U| \psi\rangle|^{2}$ for any state $\omega$. Assume that $\omega$ is $\left(a|0\rangle+\mathrm{e}^{\mathrm{i} \tau} b|1\rangle\right)$. The final step is

3) Mesure the resulting state in a basis that reproduces the desired transition probability.

For this to be possible, one has to have classical communication from the first measurement. If the first measurement projected the first qubit onto $\eta_{+}$we just measure the second qubit with $|\omega\rangle\langle\omega|$ to obtain the transition probability, but in the case $\eta_{-}$we would get wrong numbers. However, by classical communication we know which of the two cases resulted in the first measurement. If the case was $\eta_{-}$then we can measure the projector $|\tilde{\omega}\rangle\langle\tilde{\omega}|$ where $\tilde{\omega}=\left(a|0\rangle-\mathrm{e}^{\mathrm{i} \tau} b|1\rangle\right) / \sqrt{2}$. An easy calculation shows that $\left\langle\omega \mid \psi_{+}\right\rangle=\langle\omega|U| \psi\rangle=\left\langle\tilde{\omega} \mid \psi_{-}\right\rangle$hence by measure- 
ment and classical communication alone we can reproduce the matrix elements of unitary operators. Of course the above was just a sketch, but the result is general as shown by the one-way computation literature. See [1] and references therein.

Now to be sure, one has to produce the entangled state in step 1). This can be done, given $\psi$, with measurements and classical communication if one has as an initial resource a certain entangled states of several qubits. Again, see [1]. This initial entangled state as a resource for all subsequent computation steps is an earmark of this type of processing. This resource gets depleted by decoherence resulting from measurements as the computation progresses, so the process in not reversible, hence the moniker "one-way".

Now since all that any experiment does is establishes transition probabilities and one has no access to the state except through measurements, one cannot know, paraphrasing, whether what one is really measuring is $\left|\left\langle\omega \mid \psi_{+}\right\rangle\right|^{2}$ or $\left|\left\langle\tilde{\omega} \mid \psi_{-}\right\rangle\right|^{2}$ as both are equal to $|\langle\omega|U| \psi\rangle|^{2}$ which is what we are really after. One can thus ask if what is really going on in quantum experiments involving unitaries, such as time evollution, is a simulation of unitaries through some background oneway processes as exemplified by one-way quantum computation. To make this idea clearer we quote the following popular dictum of animal psychology: under rigorously controlled laboratory conditions, a rat does what it damn well pleases. Taken over to experimental physics this becomes: under rigorously controlled laboratory conditions, a measuring instrument does what it damn well pleases.

Suppose that all that exists are measurements and classical communications. As an experimentalist can prepare a state and subject it to operations and in the end set up his measuring apparatus to measure the outcome in a basis $\phi_{1}, \ldots, \phi_{n}$. Now the measuring instrument has received classical communication from past causally related events, so it does what it damn well pleases and, taking into account your settings and the communication received, actually performs a measurement in an alternate basis $\tilde{\phi}_{1}, \ldots, \tilde{\phi}_{n}$. This basis is chosen in such a way as to reproduce exactly the transition probabilities of a unitary theory. Unitary time evolution is thus subsumed under measurements and classical communication.

Does this ontology hold water? Well, to the extend that one-way computation is feasible, it does. This is already a good indication of its probable consistency. Some further considerations are still in order.

First of all, there is the initial entangled state. One has to suppose that this is the initial state of the universe. We will not go into how this state has come to be. Just as any other "singular beginning" for the universe, this is merely a hypothesis without further explanation. This initial resource gets used up as time 
progresses and the fundamental degrees of freedom of the universe get more and more disentangled. An intrinsic and natural arrow of time is part of this view.

One may wonder "what about EPR correlations"? If what we have are classical events, how can these correlations exist? Don't they now have to satisfy Bell's inequalities? Well, the state has to be created by some measurement event which may not really correspond to the EPR state the experimenter intends, by the laboratory rat principle. The information as to which state was created is now broadcast by this event. What we must not assume is that this event is "local" in the customary sense, again by the same principle. In a sense the "events" are the ingredients of an emergent space-time and for this ontology to work one has to assume that locality notions are constructed from the events, and the experimenter has no control, once more by the same principle, of what is "local" and what is not. So, the created state now suffers ostensibly a unitary time evolution which brings it to the measuring apparatus at both arms of the EPR experiment. This is done by the one-way quantum computation scheme through hidden measurement events not accessible to the experimenter. The measuring apparatus set up by the experimenter, having received all the classical information released by the creation event and the events of the ostensible unitary evolution now react, being the self-respecting laboratory rats that they are, in such a way as to reproduce exactly the EPR quantum correlations. If this is a hidden-variable theory, and it is not clear that it should be so considered, it seems it must be non-local and somewhat conspiratorial.

\section{Acknowledgements}

This research received partial financial support from the Conselho Nacional de Desenvolvimento Científico e Tecnológico (CNPq).

\section{References}

[1] Browne E. and Briegel J., One-way Quantum Computation. A Tutorial Introduction, quant-ph/0603226.

[2] Coecke B., The Logic of Entanglement, quant-ph/0402014.

[3] Svetlichny G., Tensor Universality, Quantum Information Flow, Coecke's Theorem, and Generalizations, quant-ph/0601093. 
George Svetlichny

Departamento de Matemática

Pontifícia Universidade Católica

Rio de Janeiro

BRAZIL

E-mail address: svet lich@mat.puc-rio.br 